P158 (continued)

African Americans (AA) to inform development of culturally-tailored nutrition education materials.

Study Design, Setting, Participants: The crosssectional self-administered survey was completed in face-to-face meetings with a convenience sample of AAs age 18-30 years in metro-Phoenix. Participants were recruited through local college email listservs, flyers at churches, health clinics, and by word-of-mouth. AAs have higher rates of chronic diseases in comparison to non-Hispanic Whites. Since dietary intakes influence chronic disease development, interventions for young adults may reduce future risk. Ethnic identity strongly influences dietary patterns and perceptions of health risk, but few studies have examined these associations among young adult AAs.

Outcome Measures and Analysis: The Jackson Heart Study food frequency questionnaire (FFQ) identified individual foods as well as nutrient intakes. Six subscales of ethnic identity were measured with the Multidimensional Inventory of Black Identity (MIBI) as described by Sellers 1997. Descriptive statistics, correlations, and ANOVA of dietary intakes and MIBI scores were compared.

Results: Significant differences in consumption frequencies and MIBI constructs were observed among the 203 respondents (75 men, 128 women; $22.3 \pm 3.6$ years). For example, eight foods were positively associated with the 'Nationalist' subscale (uniqueness of Black culture) green beans, peas, broccoli, corn, fried fish, juice, and fruit drinks. Other MIBI differences varied by education level with those less educated consuming more Soul Foods.

Conclusions and Implications: These results suggest ethnic identity dimensions influence dietary intakes, but in ways that are mediated by education. These factors should be considered in designing culturally tailored nutrition interventions.

Funding: None

\section{P159 Designing for Health and Appetite: Nutrition and Interior Design Professionals Create Appropriate Environments to Achieve Meal Satisfaction in Dementia Residents}

Valencia Browning-Keen, PhD, RD,LD, vbk001@shsu.edu, Sam Houston State University, Margaret Lea Houston Building, Office 225, Huntsville, TX 77341;

Kristin Kabay, MS, RD, Sam Houston State University; Leslie Boyd, MS, RD; Allison Beck, MS, RD;

Laura Burleson, PhD

Objective: To assess current practices used by facilities caring for dementia residents to determine their understanding of the benefits of appropriate foodservice environmental design which may contribute to meal satisfaction and reduce unintentional weight loss.

Study Design: One hundred and fifteen surveys were provided to administrators to assess knowledge of menu and design appropriateness of facilities to achieve meal time satisfaction.
Setting and Participants: Thirty-one facilities completed the survey, including elder residential communities, elder daycare support communities, an inpatient acute care hospital, an acute care/rehab unit and Meals on Wheels Senior Center.

Results: Fifty-five percent of participants allow their dementia residents an hour or longer to consume their meals. Seventy-one percent use plated meal service and only two facilities use family meal service. Forty-two percent of facilities play background music during periods of eating but no universal design was identified to enhance appetite in all the facilities. Only $45.2 \%$ routinely performed subjective global assessment (SGA) on their dementia residents. Conclusions and Implications: There are still gaps present in the implementation of policies of universal design that were obvious to enhance food intake and mealtime satisfaction in dementia residents. It is apparent that many facilities need to be educated on the appropriate care to enhance mealtime satisfaction and ensure that dementia residents were receiving proper nutritional care. Policies are needed for universal design to enhance dementia dining facilities. Recommendations were provided to the facilities. The survey instrument could also be improved to define design principles in detail.

Funding: None

\section{P160 Development of a Church Environment Audit Tool for Evaluating Behavioral Health Supports in Faith-Based Settings}

Nathan A. Peters, BS, npeters@email.sc.edu, University of South Carolina, 921 Assembly Street, Columbia, SC 29212; Andrew T. Kaczynski, PhD, University of South Carolina; Caroline G. Dunn, MS, LDN, RD; Danielle E. JakeSchoffman, PhD, University of Massachusetts Medical School; Sara Wilcox, PhD, University of South Carolina

Objective: To develop an environmental audit tool enabling researchers to reliably assess supports for healthy eating (HE) and physical activity (PA) within churches.

Theory, Prior Research, Rationale: Churches are key community settings that serve diverse groups frequently underrepresented in preventive health research. Recently, there has been increased attention on creating partnerships with churches for health promotion. However, audit tools to assess a church's HE/PA environment are lacking. Description: The Church Environment Audit Tool was created for use in faith-based health behavior research. The multiphase process for audit tool development included drafting an initial tool comprised of elements from audit tools used for comparable settings designed to measure similar environmental features of interest, obtaining stakeholder and expert feedback, and pilot testing in three churches in a South Carolina county. Recommendations from stakeholder and expert reviews were combined with modifications after pilot testing to create the Church Environment Audit Tool. The tool assesses support for indoor and outdoor PA, food preparation, food and beverage vending, and media such as bulletin boards and other materials about HE/PA. A script for use during administration and

Continued on page S87 